\title{
Petersen-Varchenko's Identity for Stirling Numbers of the First Kind
}

\author{
C. G. León-Vega， J. López-Bonilla， S. Yáñez-San Agustín \\ ESIME-Zacatenco, Instituto Politécnico Nacional, Edif. 5, 1er. \\ Piso, Col. Lindavista CP 07738, CDMX, México \\ Corresponding author:jlopezb@ipn.mx
}

Received: Dec 20, 2017 Revised: Jan 2, 2018 Accepted: Jan 7, 2018

\begin{abstract}
Stirling numbers of the first kind has some interesting interpretations. In this short paper, we exhibit an elementary deduction of an identity for $S_{n}^{(m)}$ obtained by Petersen-Varchenko.
\end{abstract}

Keywords: Stirling numbers of the first kind, Polynomials

\section{Introduction}

Petersen-Varchenko [7] proved the following identity:

$$
S_{n}^{(n-k)}=\frac{1}{k} \sum_{m=1}^{k}(-1)^{m}\left(\begin{array}{c}
m+n-k \\
m+1
\end{array}\right) S_{n}^{(m+n-k)}, \quad 1 \leq k \leq n,
$$

for the Stirling numbers of the first kind [9]. On the other hand, we know the expression $[1,3,8]$ :

$$
S_{n}^{(n-k)}=\sum_{j=n-k-1}^{n-1}(-1)^{n-k+j-1}\left(\begin{array}{c}
j \\
n-k-1
\end{array}\right) S_{n-1}^{(j)} .
$$

In Section 2, we show that (2) implies (1).

\section{Petersen-Varchenko's Formula}

From (2), we have

$$
\begin{array}{r}
S_{n}^{(n-k)}=-\sum_{m=-1}^{k-1}(-1)^{m}\left(\begin{array}{c}
m+n-k \\
m+1
\end{array}\right) S_{n-1}^{(m+n-k)} \\
=-\sum_{m=1}^{k-1}(-1)^{m}\left(\begin{array}{c}
m+n-k \\
m+1
\end{array}\right) S_{n-1}^{(m+n-k)}+S_{n-1}^{(n-k-1)}-(n-k) S_{n-1}^{(n-k)},
\end{array}
$$

and in the left side of (3) we employ the recurrence [8]

$$
S_{n}^{(n-k)}=S_{n-1}^{(n-k-1)}-(n-1) S_{n-1}^{(n-k)},
$$


thus

$$
(k-1) S_{n-1}^{(n-k)}=\sum_{m=1}^{k-1}(-1)^{m}\left(\begin{array}{c}
m+n-k \\
m+1
\end{array}\right) S_{n-1}^{(m+n-k)},
$$

where we make the changes $n \rightarrow n+1$ and $k \rightarrow k+1$ to obtain (1), QED.

Now we shall deduce an alternative relation to (1); in fact, from $[2,4]$ we have the expression:

$$
S_{n}^{(n-k)}=\left(\begin{array}{c}
n-1 \\
k
\end{array}\right) B_{k}^{(n)},
$$

with the participation of the Nörlund polynomials $B_{m}^{(z)}[5]$, that is,

$$
S_{n}^{(j)}=\left(\begin{array}{c}
k+j-1 \\
k
\end{array}\right) B_{k}^{(k+j)} .
$$

Besides in $[2,5]$, we find the property:

$$
\begin{aligned}
& (-1)^{k}\left(\begin{array}{l}
Z \\
k
\end{array}\right) B_{k}^{(k-z)}=\sum_{j=0}^{k}\left(\begin{array}{c}
k+j-1 \\
k
\end{array}\right)\left(\begin{array}{c}
k-z \\
k+j
\end{array}\right)\left(\begin{array}{l}
k+z \\
k-j
\end{array}\right) B_{k}^{(k+j),} \\
& \Rightarrow B_{k}^{(n)} \stackrel{(5)}{=} \frac{(-1)^{k}}{\left(\begin{array}{c}
k-n \\
k
\end{array}\right)} \sum_{j=0}^{k}\left(\begin{array}{c}
n \\
k+j
\end{array}\right)\left(\begin{array}{c}
2 k-n \\
k-j
\end{array}\right) S_{n}^{(j)}
\end{aligned}
$$

then from (4) and (7) we obtain the following identity for Stirling numbers of the first kind:

$$
S_{n}^{(n-k)}=\sum_{j=1}^{k}\left(\begin{array}{c}
n \\
k+j
\end{array}\right)\left(\begin{array}{c}
2 k-n \\
k-j
\end{array}\right) S_{k+j}^{(j)}, \quad 1 \leq k \leq n,
$$

which if $k=1,2$ gives the known results [3]:

$$
S_{n}^{(n-1)}=-\left(\begin{array}{l}
n \\
2
\end{array}\right), \quad S_{n}^{(n-2)}=\frac{1}{4}\left(\begin{array}{l}
n \\
3
\end{array}\right)(3 n-1) .
$$

It is possible to construct many identities for $S_{n}^{(m)}$, for example, from [3], we have:

$$
(n-k) S_{n}^{(k)}=\sum_{j=k+1}^{n}(-1)^{k+j}\left(\begin{array}{c}
j \\
k-1
\end{array}\right) S_{n}^{(j)},
$$

and from $[8]$

$$
\frac{(-1)^{n}}{n !} \sum_{j=k-1}^{n}(-1)^{k+j-1}\left(\begin{array}{c}
j \\
k-1
\end{array}\right) S_{n}^{(j)}=\sum_{r=0}^{n} \frac{(-1)^{r}}{r !} S_{r}^{(k-1)},
$$

we deduce the following relation

$$
S_{n+1}^{(k)}=(-1)^{n} n ! \sum_{m=0}^{n} \frac{(-1)^{m}}{m !} S_{m}^{(k-1)} .
$$




\section{Conclusion}

Petersen-Varchenko [7] obtain formulas for the growth rate of the number of certain paths in a multi-dimensional analogue of the Eulerian graph, and as a corollary they deduce the identity (1). Our approach shows that (1) can be proved via an elementary process.

\section{References}

[1] Benjamin AT, Preston GO and Quinn JJ (2002), A Stirling encounter with harmonic numbers, Maths. Mag., 75(2) : 95-103.

[2] Carlitz L (1960), Note on Nörlund polynomials $\mathrm{B}_{\mathrm{n}}^{(\mathrm{z})}$, Proc. Am. Math. Soc., 11(3) : 452-455.

[3] Comtet L (1974), Advanced combinatorics, D. Reidel Pub, Dordrecht, Holland.

[4] Gessel IM (2005), On Miki's identity for Bernoulli numbers, J. Number Theory, 110(1) : 7582.

[5] Gould HW (1960), Stirling number representation problems, Proc. Am. Math. Soc., 11(3) : 447-451.

[6] Nörlund NE (1924), Vorlesungen über differenzenrechnung, Springer-Verlag, Berlin.

[7] Petersen K, Varchenko A (2012), Path count asymptotics and Stirling numbers, Proc. Am. Math. Soc., 140(6) : 1909-1919.

[8] Quaintance J and Gould HW (2016), Combinatorial identities for Stirling numbers, World Scientific, Singapore.

[9] Riordan J (1968), Combinatorial identities, John Wiley \& Sons, New York. 